\title{
Cardiac-Activity Measures for Assessing Airport Ramp-Tower Controller's Workload
}

Miwa Hayashi, NASA Ames Research Center

Victoria Dulchinos, San Jose State University Foundation

Human Factors and Ergonomics Society (HFES) Annual Meeting

September 19-23, 2016, Washington, DC 


\section{Background}

- Subjective measures of workload have known shortcomings.

- "Subjective"

- Low sensitivity 


\section{Low Sensitivity}

"Please rate your workload level on the scale of 1 to 7 , where 1 is the lowest workload and 7 is the highest."

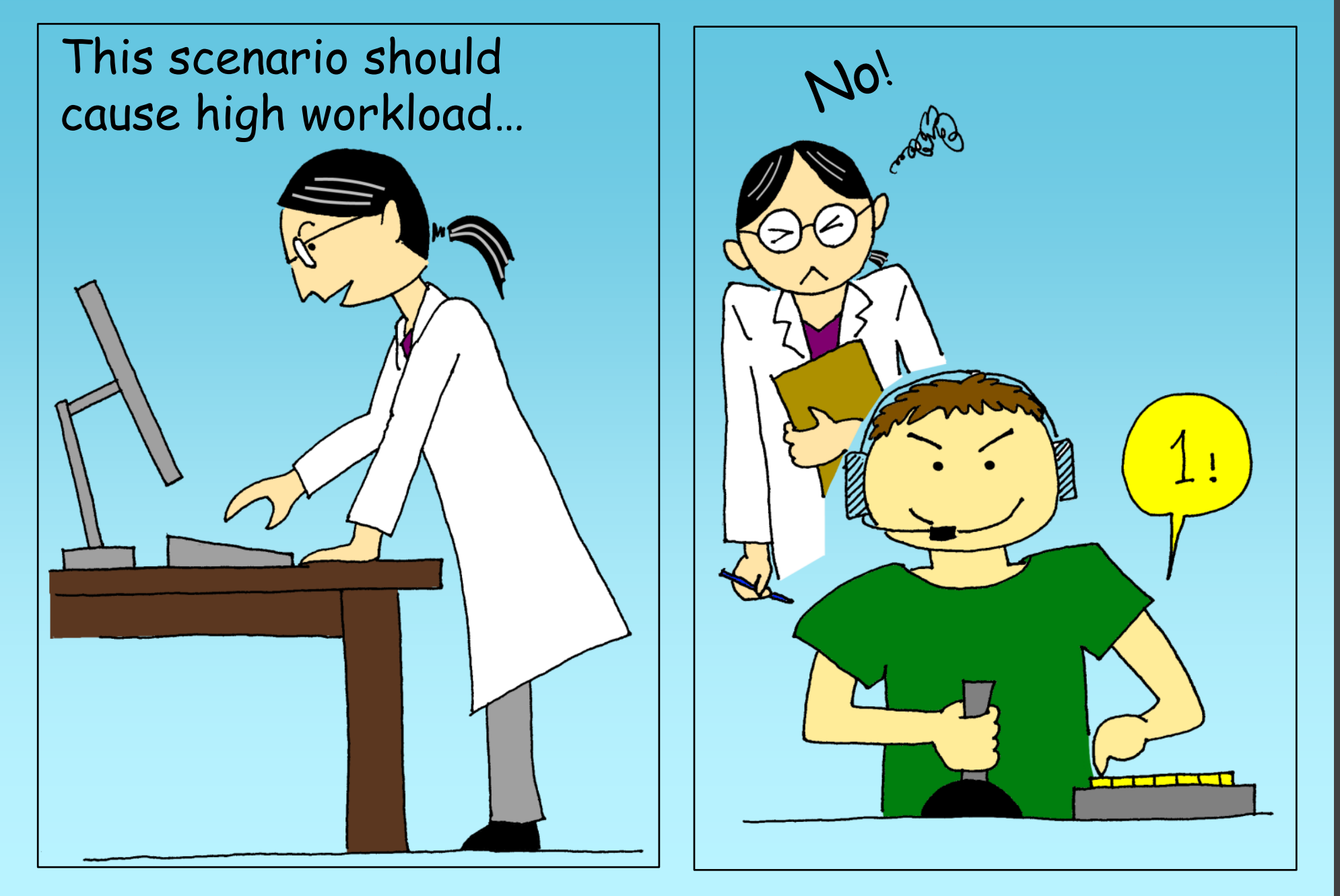


Another case of low sensitivity
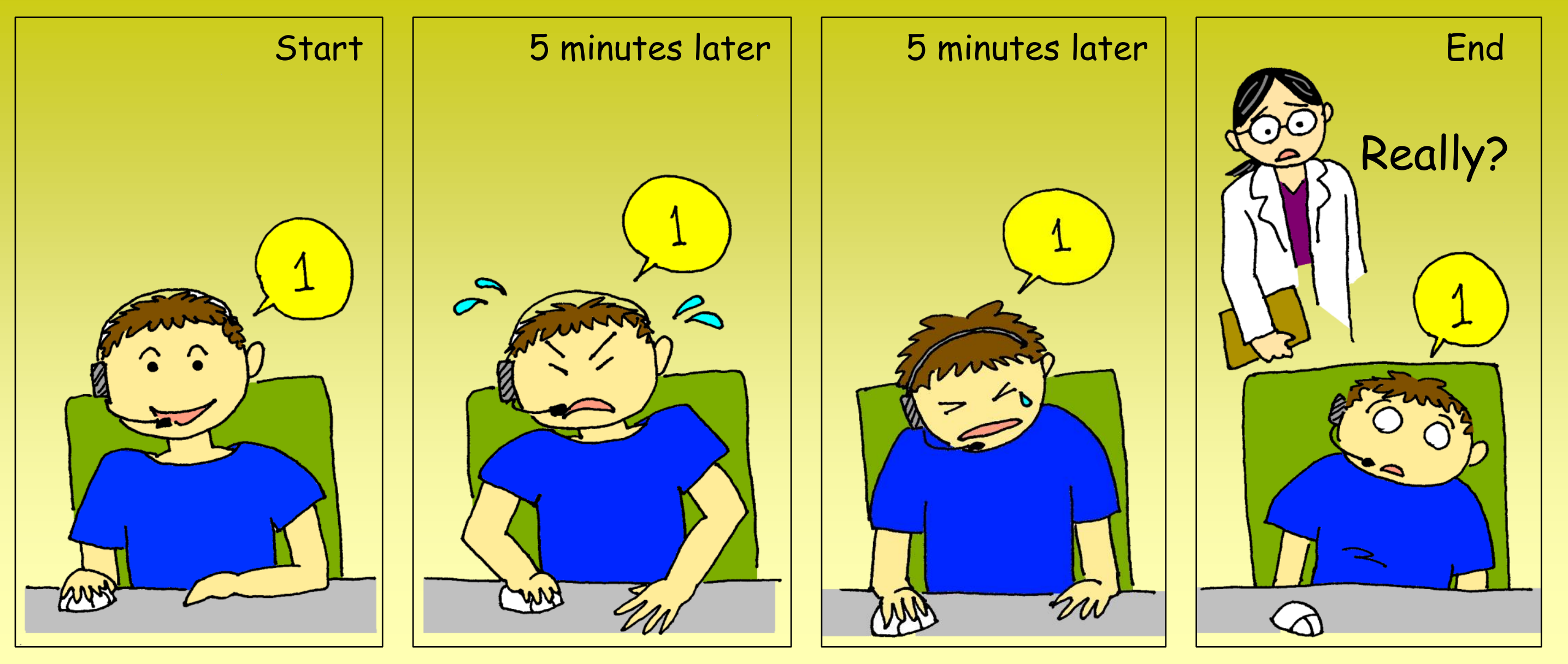

(C) 2016 Miwa Hayashi 


\section{Background}

- Subjective measures of workload have known shortcomings.

- "Subjective"

- Low sensitivity

- Sparse data, likely missing the important event

- Potentially distracting, if measured in real time
"Objective"

"??"
"Continuous"

"Non-intrusive"

- Physiological measures may potentially address these weaknesses. 


\section{Goal}

To examine if mean heart rate (HR) and heart rate variability (HRV) can be used to measure the controller workload in our airtraffic-control simulation evaluation studies.

- Compared mean HR and HRV with the real-time selfreported subjective workload rating results.

- Assessed if mean HR and HRV could replace the subjective measures (e.g., in field tests). 


\section{Mean Heart Rate (HR)}

- The average number of beats/minute.

- Derived from the "RR intervals."

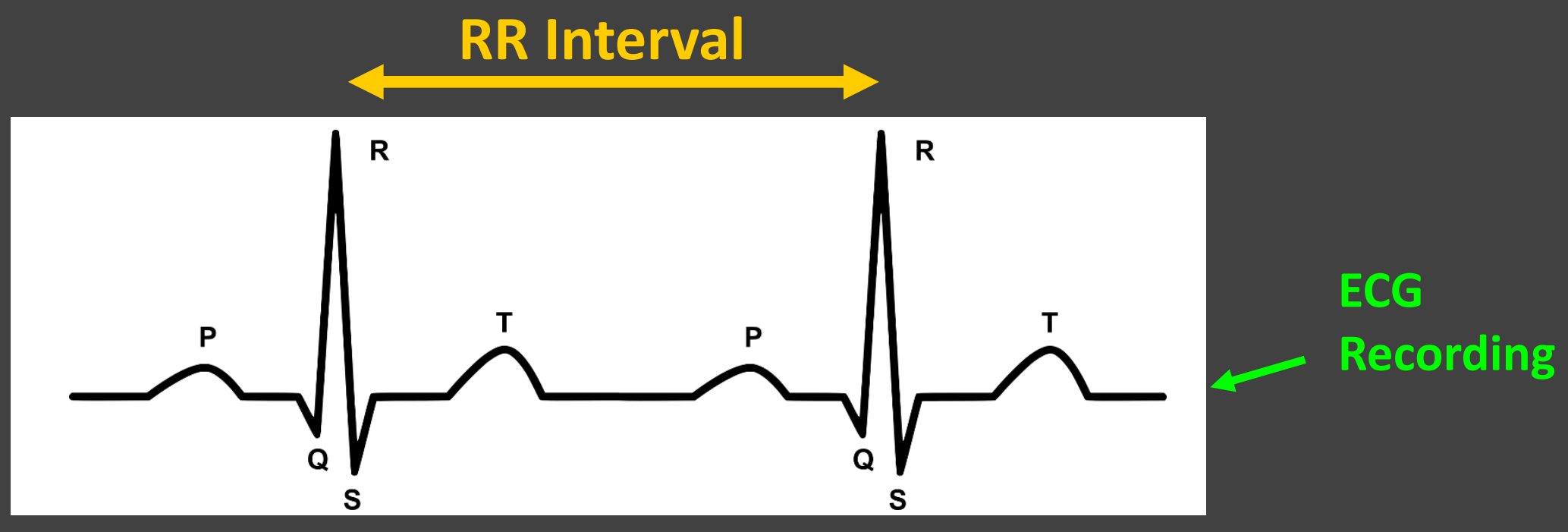

Credit: Agateller (Anthony Atkielski) via Wikimedia Commons

- Considered to reflect an overall level of general arousal, physical work, task demands, and emotional response. (Wierwille \& Eggemeier, 1993) 


\section{Heart Rate Variability (HRV)}

- Measure of variability in the RR intervals.

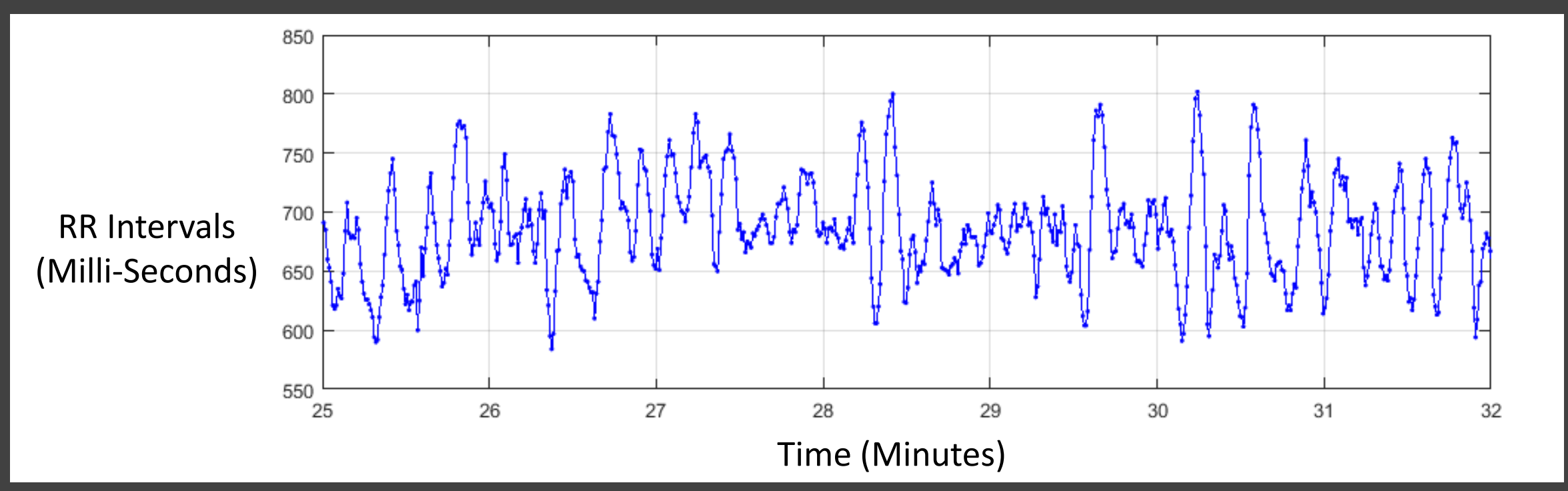

- Thought to reflect the balance of autonomic nervous system:

- HF power $(0.15-0.4 \mathrm{~Hz})$ : Parasympathetic activity

- LF power (0.04-0.15 Hz): Parasympathetic and sympathetic activities

- MF power suppression $(0.08-0.15 \mathrm{~Hz})$ : Increased cognitive effort 


\section{Past Studies}

- HRV MF suppression used to measure workload:

\begin{tabular}{ll}
\hline \multicolumn{1}{c}{ Authors (Year) } & \multicolumn{1}{c}{ Studied Task } \\
\hline Vicente, Thornton, \& Moray (1987) & Low-fidelity hovercraft course-tracking simulation \\
Rowe, Sibert, \& Irwin (1998) & Air-traffic-control game \\
Tattersall \& Hockey (1995) & Military long-haul flight simulation \\
\hline
\end{tabular}

- Skeptics:

- Inconsistent MF results for AGARD-STRESS battery task workload (Nickel \& Nachreiner, 2003)

- Large individual differences in stress reactions of autonomic nervous system (Berntson \& Cacioppo, 2004) 
Methods 


\section{Airport Ramp Tower Simulation}

- Simulation evaluation of a NASA's departure-metering decision-support tool, Spot and Runway Departure Advisor (SARDA).

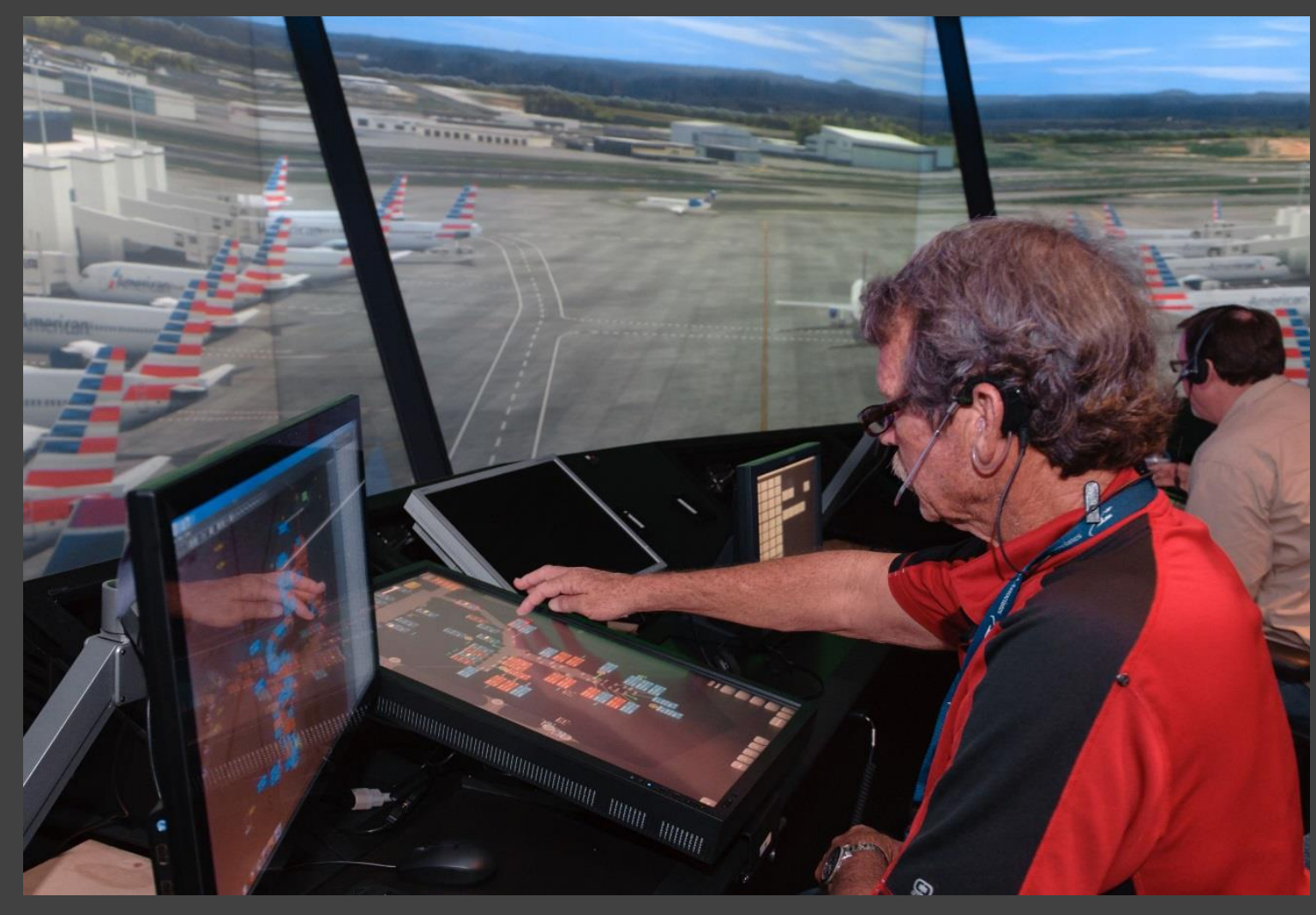

- 6 Charlotte airport ramp-tower controllers

- 16 runs per controller

- 65-70 minutes departure "push"

- Self-reported subjective workload rating at every 5 minute

- Resulted in $10-12 \%$ taxi fuel saving

- No increase in the controller workload 


\section{ECG Recording}

- RR intervals were recorded with Firstbeat Bodyguard 2 (BG2).

- Attached to the body via 2 electrodes.

- Sampling rate $=1000 \mathrm{~Hz}$

- No activity constraint

- Free to sit, stand, and walk around.

- OK to smoke or drink tea/coffee
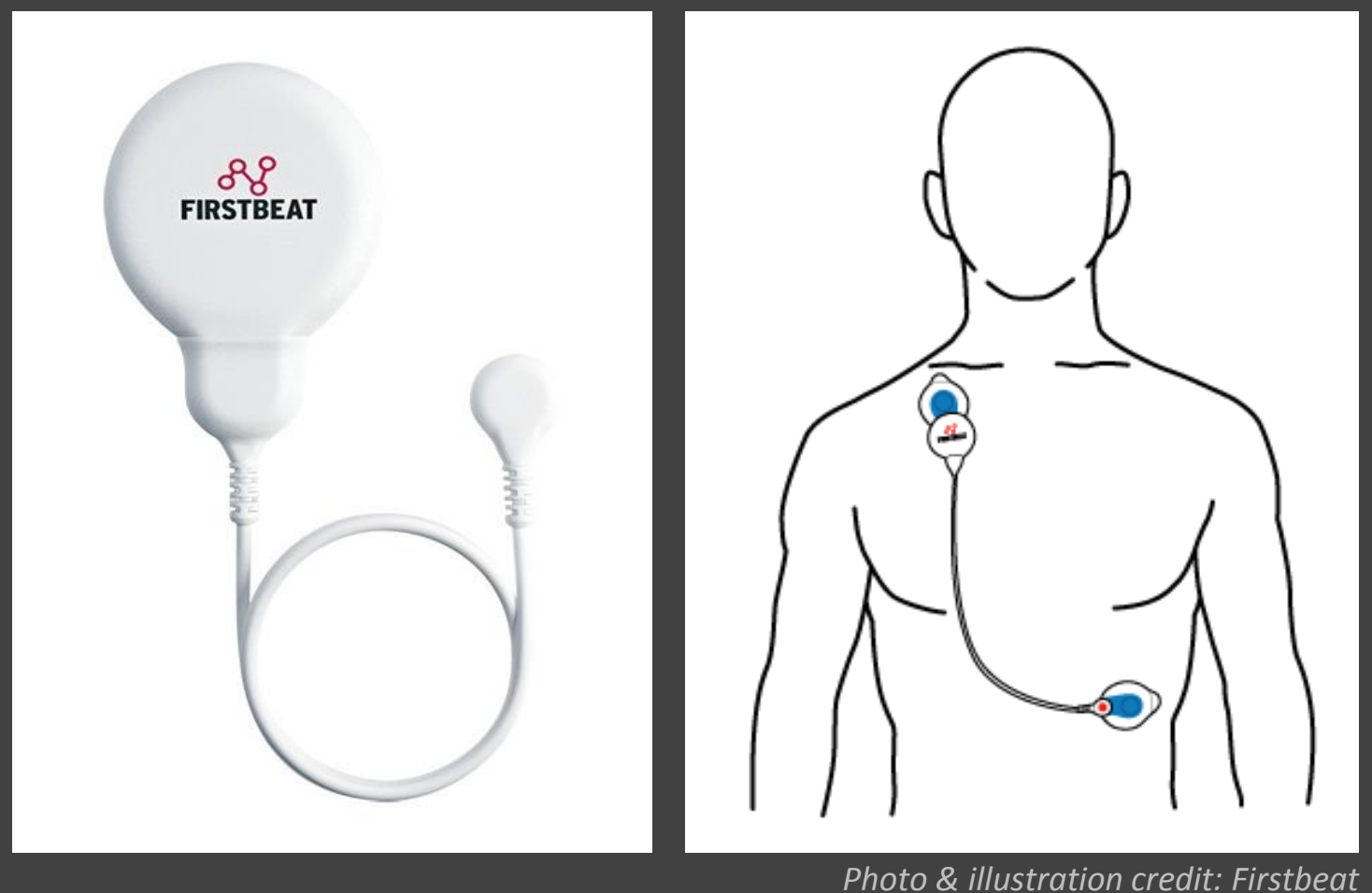
during a break. 


\section{Computation of Mean HR and HRV}

1. Artifacts in the RR interval data were removed. (No replacement.)

2. Mean HR were computed within the 2-minute windows around the sampling times of the real-time workload ratings.

3. Within the same 2-minute windows, MF, HF, and the total power (0.04 - $0.15 \mathrm{~Hz}$ ) HRV were computed.

- Lomb-Scargle Periodogram algorithm was used to estimate the power spectral density.

4. MF and HF were normalized with the total power. 


\section{Statistical Tests}

Linear Mixed Model (LMM) regression was applied.

Mean HR, HRV MF (normalized), or HRV HF (normalized)

Workload rating

- Fixed, continuous effect
$W L+P+W L^{*} P+\varepsilon$

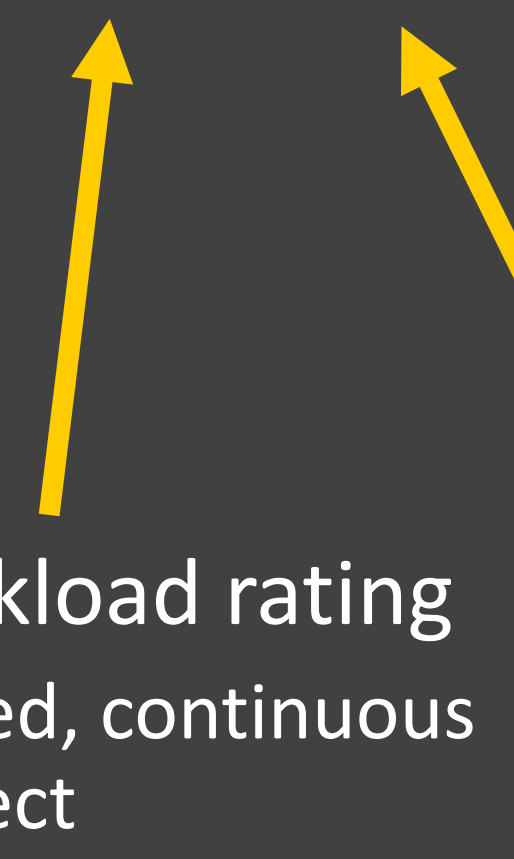

Participant effect

\section{Error} Interaction

- Random, categorical 
Results \& Discussion 


\section{Mean HR Results}

- The LMM did not find statistical significance in workload (WL) effect.

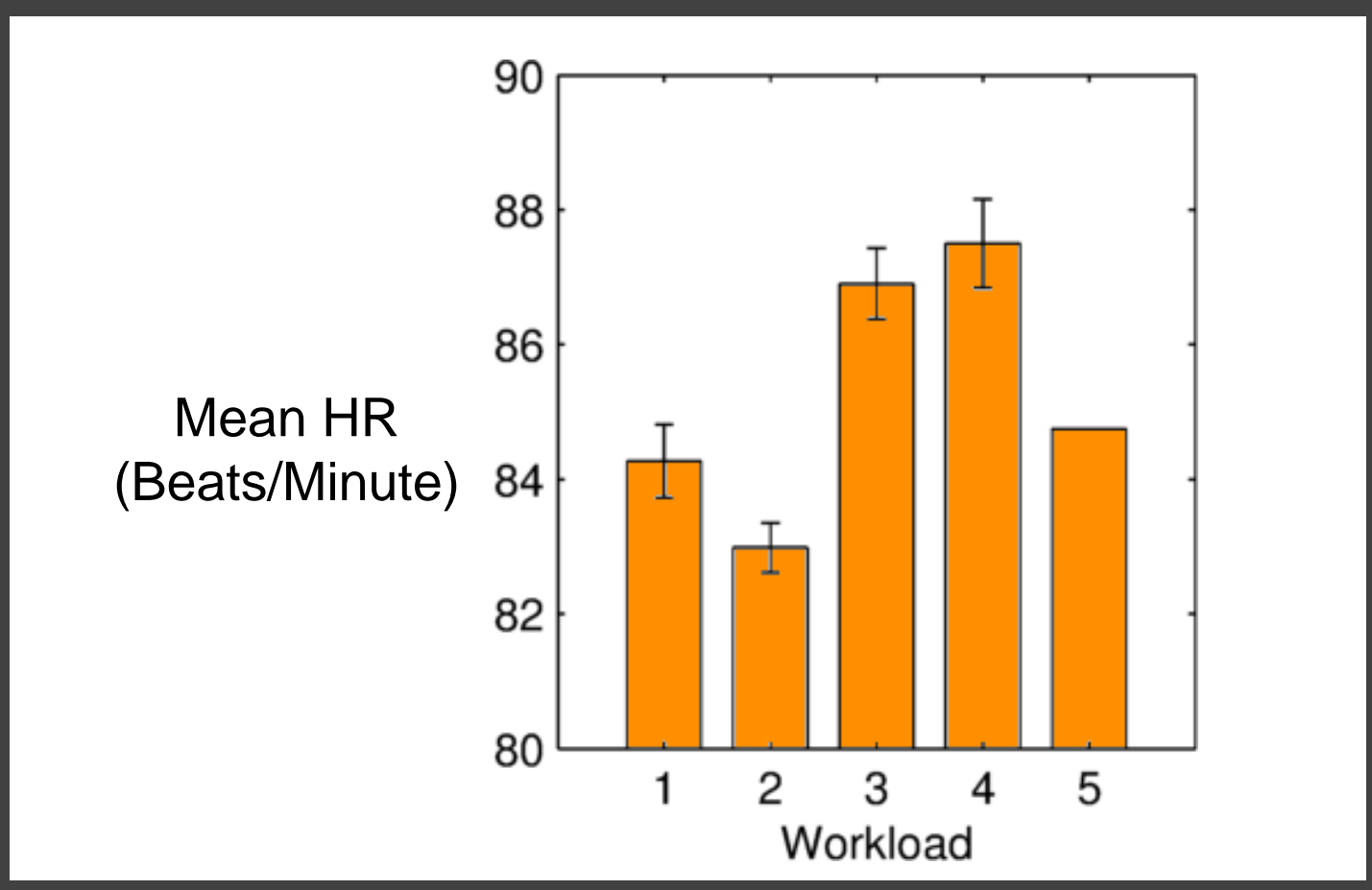

- The graph shows only a weak trend. 


\section{HRV Results}

- For the normalized MF, WL effect was statistically significant $(p<0.01)$.

- However, the estimated coefficient was in the wrong direction $(0.015 ; S E=0.006)$.
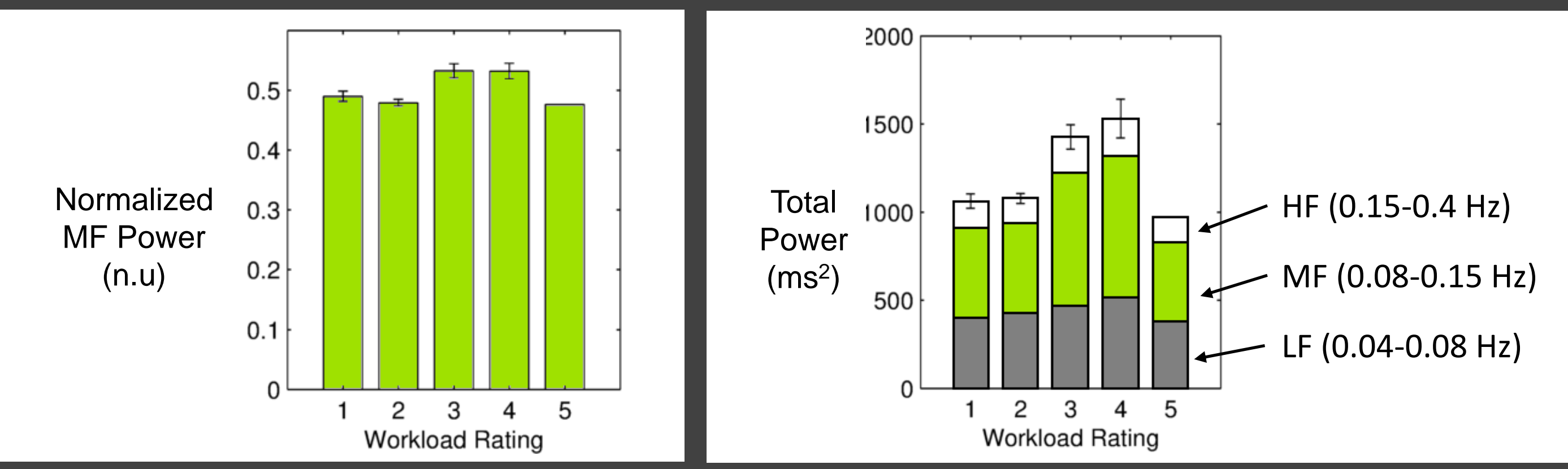

- The total power also increased when $\mathrm{WL}=3$ or 4 (again, the wrong direction). 


\section{Discussion}

- Mean HR: only weak correlation with the workload ratings

- Subjective measures are not necessarily the true state of workload.

- HRV-MF and HRV-Total power: contradictory trend directions

- Were they more "relaxed" when they reported WL $=3$ or 4 ? (Unlikely.)

- More plausible explanation: HRV must have sensed something else.

- Increased speech when traffic volume was high.

- Posture change, walking around, sipping water, etc. 


\section{Summary}

- In our airport ramp-tower simulation, we found that mean HR was only weakly correlated with the controller's self-reported workload levels.

- HRV results were contradictory and inconclusive.

- Until further research is conducted to understand the effects of speech, posture changes, etc., using HR or HRV measures as a sole mean of workload assessment in field tests is not recommended.

- It is recommended to measure HR and HRV along with subjective measures. 